\title{
Essais
}

ESSAIS

Revue interdisciplinaire d'Humanités

$14 \mid 2018$

Plurilinguismes en construction

\section{Le poids des mots pour trouver sa place dans l'espace scolaire}

\section{Catherine Mendonça Dias}

\section{OpenEdition}

1 Journals

Édition électronique

URL : http://journals.openedition.org/essais/323

DOI : 10.4000/essais.323

ISSN : 2276-0970

Éditeur

École doctorale Montaigne Humanités

\section{Édition imprimée}

Date de publication : 1 juin 2018

Pagination : 67-95

ISBN : 979-10-97024-06-2

ISSN : 2417-4211

\section{Référence électronique}

Catherine Mendonça Dias, «Le poids des mots pour trouver sa place dans l'espace scolaire », Essais [En ligne], 14 | 2018, mis en ligne le 01 décembre 2019, consulté le 12 décembre 2019. URL : http:// journals.openedition.org/essais/323; DOI : 10.4000/essais.323 


\section{Le poids des mots pour trouver sa place dans l'espace scolaire}

\section{Catherine Mendonça Dias}

Au moins 37200 élèves allophones sont arrivés en France au cours de l'année scolaire 2014-2015 . Enfants ou adolescents, ils ont été amenés à quitter leur pays et les voici désormais en France, devant poursuivre leur scolarité avec une langue inconnue ou peut-être familière suivant qu'ils sont issus de pays ou de cellules familiales francophones ${ }^{2}$, ou qu'ils ont étudié précédemment le français en tant que langue vivante.

En fonction de leurs besoins d'apprentissage et des capacités d'accueil des dispositifs, certains jeunes rejoignent des Unités Pédagogiques pour élèves Allophones Arrivants (UPE2A) au sein desquelles ils bénéficient d'un apprentissage intensif du français dans lequel " la langue française n'y est pas seulement objet d'étude mais aussi outil d'apprentissage des autres objets d'étude $»^{3}$, suivant une didactique et une pédagogie facilitant l'accès aux compétences visées par leur cycle. Cette UPE2A n'est pas régie par un programme, particularité singulière dans le paysage scolaire français, comme nous l'avions déjà souligné dans une recherche antérieure ${ }^{4}$, et un seul référentiel officiel a été publié en $2000^{5}$ pour spécifier l'organisation de l'enseignement, initialement référé comme situation d'enseignement $\mathrm{du}$ " français langue seconde ${ }^{6}$ ou encore,

1 Juliette Robin et Mustapha Touahir, "Année scolaire 2014-2015 : 52500 élèves allophones scolarisés dont 15300 l'étaient déjà l'année précédente ", Note d'information n 35, octobre 2015, Direction de l'Évaluation de la Prospective et de la Performance.

2 Jean-Pierre Cuq, Le Français Langue Seconde. Origines d'une notion et implications didactiques, 1991, Paris, Hachette.

3 Catherine Marcus, Français Langue Seconde, lectures pour le collège, CDRP de l'Académie de Grenoble, La collection 36, Delagrave, 1999, p. 12.

4 Catherine Mendonça Dias, Les progressions linguistiques des collégiens nouvellement arrivés en France, Villeneuve d'Ascq, Publication ANRT, 2012.

5 Bertrand Denis, Alain Viala, Gérard Vigner (éds), Le Français Langue Seconde, Centre National de Documentation Pédagogique (CNDP), Collège-repères, publication du Ministère de l'Éducation Nationale, 2000.

6 Gérard Vigner, Le français langue seconde, comment apprendre aux élèves nouvellement arrivés. Paris, Hachette Éducation, 2009. 
en tant que "langue de scolarisation ${ }^{7}$ où l'objectif d'apprentissage du français pourrait être concomitant avec le développement de compétences plurilingues, en s'appuyant sur des approches plurielles, par exemple à travers la comparaison des langues?. Plus récemment une collection est parue intitulée "Cap sur le français langue de scolarisation ${ }^{10}$, pour apporter des repères aux enseignants. Quelques recherches universitaires récentes ont apporté un éclairage sur différentes disciplines étudiées en langue seconde : la didacticienne Fatima DavinChnane a mis en évidence la nécessité de ralentissement didactique ${ }^{11}$ tandis que pour les mathématiques, Karine Millon-Fauré a analysé les liens entre la communication usuelle et la langue de spécialité ${ }^{2}$ ou encore, Elisabeth Faupin s'est interrogée sur la production orale à travers les disciplines scolaires ${ }^{13}$.

Quand bien même les élèves sont inscrits pédagogiquement dans une UPE2A, ils suivent aussi un cycle régulier, dans une classe ordinaire, telle que les politiques inclusives le recommandent malgré des obstacles qui ne manquent pas de se trouver ${ }^{14}$ quelles que soient les académies ${ }^{15}$. Où qu'ils se trouvent, les exigences multiples scolaires, disciplinaires, langagières, sociales... se déploient autour d'eux. Être nouveau parmi d'autres nouveaux, où l'ailleurs banalisé s'est fondu dans le quotidien de l'établissement, ce n'est pas évident surtout si l'on tend à aller vers l'autre, avec une langue véhiculaire balbutiante qui impatiente parfois l'interlocuteur. Comment les élèves, engagés dans une trajectoire migratoire intime, se doteront-ils de compétences linguistiques suffisantes pour évoluer de façon autonome dans un nouvel espace scolaire où il leur faut trouver leur place?

7 Michèle Verdelhan-Bourgade, Le français de scolarisation, pour une didactique réaliste, 2002, PUF.

8 Se reporter au cadre de référence pour les approches plurielles des langues et des cultures disponible sur : http://carap.ecml.at/.

9 Nathalie Auger, Comparons nos langues. Démarche d'apprentissage du français auprès d'Enfants Nouvellement Arrivés (ENA), [DVD vidéo], Montpellier, CRDP Académie de Montpellier, 2005.

10 Catherine Klein (éd.), Le français comme langue de scolarisation. Accompagner, enseigner, évaluer, se former, Collection Cap sur le français de la scolarisation, Scéren, CNDP-CRDP, 2012.

11 Fatima Davin-Chnane, Didactique du FLS en France : le cas de la discipline "français » enseignée au collège, Villeneuve d'Ascq, publication ANRT, 2005.

12 Karine Millon-Fauré, Les répercussions des difficultés langagières des élèves sur l'activité mathématique en classe : le cas des élèves migrants. Thèse de doctorat, sous la direction d'Alain Mercier, Université Aix-Marseille I, 2011.

13 Elisabeth Faupin, Prendre la parole en classe : une gageure pour les élèves allophones arrivants : le cas des cours de français, mathématiques et histoire-géographie, Doctorat en sciences du langage, Jean-Pierre Cuq (éd.), 2015. Voir aussi Faupin ici même.

14 Guy Cherqui, Fabrice Peutot, Inclure : français de scolarisation et élèves allophones, Paris, Hachette FLE, 2015.

15 Delphine Guédat-Bittighoffer, Les élèves allophones à l'épreuve de l'apprentissage d'une langue seconde : des politiques éducatives au processus de compréhension, Doctorat en sciences du langage, Frédéric Tupin et Marie-Ange Dat (éds), Université de Nantes, 2014. 
Pour apporter quelques éléments de réponses, nous aborderons trois aspects. D'abord, nous allons définir le champ de notre public dont les circonscriptions ont varié dans le temps : c'est pourquoi nous nous intéresserons aux terminologies à travers lesquelles l'institution a construit des représentations portant sur des espaces scolaires alloués aux élèves arrivants. Puis, nous examinerons les processus par lesquels des facteurs, extérieurs ou en réaction aux conditions de scolarisation, peuvent freiner l'implication de l'enfant ou de l'adolescent dans son apprentissage de la langue française alors que celle-ci devrait faciliter sa socialisation dans l'espace scolaire. Enfin, il s'agira d'évoquer le moment à partir duquel l'élève acquiert une autonomie en langue française pour communiquer librement dans son milieu scolaire. Pour faire apparaitre des rythmes d'acquisition, nous présenterons des performances en français des élèves pris dans une dynamique d'acquisition. En écho à cette analyse, nous mettrons en lien nos conclusions avec les résultats en cours d'analyse du projet de recherches EVASCOL $^{16}$, financé par le Défenseur Des Droits et porté par le GRAHPES ${ }^{17}$, dont les résultats concourront à caractériser la scolarisation des élèves allophones arrivants, tout en prenant compte les aménagements spécifiques dans la prise en charge pédagogique et les performances linguistiques et scolaires.

\section{Les implications des terminologies associées aux élèves}

La caractérisation des élèves aujourd'hui désignés comme " allophones arrivants " a évolué à travers les terminologies, porteuses d'enjeux pour leur prise en charge pédagogique spécifique. La circulaire de $1970^{18}$ initie la mise en place officielle de dispositifs ; Claude Cortier nous en rappelle ici le contexte :
«À partir des années 1970, après le choc pétrolier et la crise économique qui s’en est suivi, la migration des travailleurs étrangers est progressivement stoppée tandis que l'on organise le dispositif légal de regroupement familial. C'est ainsi que, d'une immigration de travailleurs adultes, on passe progressivement à une immigration familiale. Dans le même temps, la réglementation scolaire commence à prendre en compte de façon spécifique les enfants étrangers, et à "déroger" ainsi d'une certaine façon aux principes républicains, notamment celui de l'égalité de traitement de tous les enfants, codifiés par la loi de 1882 sur l'obligation scolaire $»^{19}$.

16 EVASCOL, "Étudier, voir et analyser la scolarisation des enfants migrants et itinérants" (https:// evascol.hypotheses.org) est une recherche financée par le Défenseur Des Droits, portée par le GRHAPES de l'INSHEA avec la coordination par Maïtena Armagnague et Isabelle Rigoni et soutenue par Claire Cossée, Catherine Mendonça Dias et Simona Tersigni, membres de l'équipe pluridiscipinaire de coordination scientifique.

17 Groupe de recherche sur le handicap, l'accessibilité et les pratiques éducatives et scolaires (EA 7287 Grhapes)

18 Circulaire n ${ }^{\circ}$ IX 70-37 du 13 janvier 1970, Classes expérimentales d'initiation pour enfants étrangers.

19 Claude Cortier, "Dispositifs et classes pour les nouveaux arrivants allophones ", in Diversité, $\mathrm{n}^{\circ} 153$, été 2008. Le principe d'hospitalité, 2008, p. 15-16. 
Les flux migratoires varient, la société et les projets de société aussi. Les mots portent une charge sémantique dont la connotation évolue dans le temps. Il a été nécessaire de nommer pour désigner et organiser l'enseignement en réponse à des besoins identifiés par des équipes éducatives. En nous interrogeant rétrospectivement sur l'évolution terminologique à travers les circulaires ${ }^{20}$, comme Gabrielle Varro ${ }^{21}$ avec son regard de sociologue ou Stéphanie Galligani ${ }^{22}$ en sciences du langage et didactique des langues, nous cherchons pour notre part à définir notre public tout en faisant apparaitre les représentations institutionnelles qui ont été construites sur ces élèves arrivants et qui motivent les orientations pédagogiques. Et comme l'écrivait Michèle Verdelhan-Bourgade, qui a contribué au développement du concept de français langue de scolarisation : "Point n'est besoin d'être linguiste averti pour comprendre l'importance de la désignation d'un objet : le nom donné ne sert pas seulement à repérer l'élément, à le cerner dans l'espace et le temps; il lui affecte une dimension, une valeur, parfois politique, parfois affective. $»^{23}$

\section{Des terminologies juridiques et sociales}

De $1936^{24}$ à $1986^{25}$, les textes officiels citent les élèves " étrangers ". Pourtant, un grand nombre d'arrivants est français, par filiation ou de naissance dans le cas du retour d'expatriées ${ }^{26}$. À titre d'exemple, en $2000^{27}$, les classes d'initiation comportaient $30 \%$ d'élèves de nationalité française. Inversement, une majorité d'élèves de nationalité étrangère, nés sur le sol français de parents étrangers ou installés en France très jeunes, ont effectué toute leur scolarité obligatoire en France ${ }^{28}$. Ainsi, le critère de la nationalité est inopérant et révèle une méconnaissance du groupe d'élèves pris en charge.

20 http://www.francaislangueseconde.fr/recherches-sur-le-fls/textes-officiels/.

21 Gabrielle Varro, "La désignation des élèves étrangers dans les textes officiels », Mots, décembre $1999, n^{\circ} 61$, p. 49-66.

22 Galligani Stéphanie, "L'identification de "l’enfant étranger" dans les circulaires de l'Éducation nationale depuis 1970. Vers la reconnaissance d'un plurilinguisme ? ", in Pierre Martinez, Danièle Moore et Valérie Spaëth (éds), Plurilinguismes et enseignement. Identités en construction. Paris : Riveneuve Éditions, 2008, p. 113-126.

23 Verdelhan-Bourgade, op. cit., p. 13.

24 Loi du 9 août 1936, Journal Officiel du 13 août 1936.

25 Circulaire no 86-120 du 13 mars 1986, Accueil et intégration des élèves étrangers dans les écoles, collèges et lycées.

26 Claire Schiff (éd.), Non scolarisation, déscolarisation et scolarisation partielle des migrants, Ministère de l'Éducation Nationale. Programme interministériel de recherche sur les processus de déscolarisation, 2003, Paris.

27 Ministère de l'Éducation Nationale, Repères et références statistiques sur les enseignements, la formation et la recherche, Paris: DEPP, 2006, p. 84-85.

28 Ministère de l'Éducation Nationale, Repères et références statistiques sur les enseignements, la formation et la recherche, Paris: DEPP, 2008, p. 29 et p. 135 : pour le second degré en 2008-2009 on recense 163881 étrangers alors que les élèves nouvellement arrivés sont estimés à 17765 . 
Dans la circulaire de 1976, on privilégie l'appellation d'" enfants de migrants ". Le nom d'" enfant " efface le degré de xénité et ramène à l'étymologie d' "infans", celui " qui ne parle pas " ${ }^{29}$. Ces enfants sont considérés par rapport au projet migratoire des parents, ce qui soulève un autre besoin supposé dans le cas d'une migration économique temporaire : celui de poursuivre l'apprentissage de la langue d'origine en vue du retour au pays, d'où la mise en place d'un enseignement de la langue et de la culture d'origine (ELCO) initié avec le portugais en $1973^{30}$, pointé du doigt par le Haut Conseil à l'Intégration en $2011^{31}$ et restructuré en Enseignements Internationaux de Langues Étrangères (EILE) depuis $2016^{32}$.

La désignation concurrente d'enfants de " travailleurs immigrés » est aussi utilisée. Remarquons qu'aujourd'hui, l'expression se révèlerait inappropriée au vu des nombreux demandeurs d'asile (qui, de par leur statut, ne sont pas autorisés à travailler sur le sol français tant que dure la procédure et qu'ils ne sont pas reconnus réfugiés) et des jeunes mineurs non accompagnés (MNA), arrivant sans parent en France ${ }^{33}$ et pris en charge par l'Aide Sociale à l'Enfance (ASE) : les exilés qui fuient une situation tragique, les mandatés envoyés par la famille notamment pour continuer leurs études, les exploités victimes de la traite, les fugueurs et les errants ${ }^{34}$. D'autres enfants encore viennent rejoindre un membre de la famille. Et certains d'arriver simplement pour un échange scolaire.

Par ailleurs, pour revenir au-delà de 1970, du parent travailleur à l'enfant ouvrier, le glissement a parfois cours si l'on en croit la journaliste Dalila Kerchouche qui, dans le roman documentaire portant sur sa famille, raconte qu’à la fin des années 60, en Lot-et-Garonne, "[les petits harkis] n'ont parfois que deux heures d'enseignement par jour. Les élèves suivent des cours de maths et de français le matin, et, l'après-midi, ils font du sport et des

29 Véronique Castellotti, La langue maternelle en classe de langue étrangère, Paris, CLE international, 2007, p. 50.

30 Circulaire $\mathrm{n}^{\circ} 73-10008$ du 2 février 1973, Enseignement du portugais à l'intention des élèves portugais scolarisés dans l'enseignement élémentaire.

31 Haut Conseil à l'Intégration, Les défis de l'intégration à l'école et Recommandations du Haut Conseil à l'intégration au Premier ministre relatives à l'expression religieuse dans les espaces publics de la République, Rapport au Premier ministre pour l'année 2010, collection des rapports officiels, p. 23 à 29 et p. 147. La recommandation $n^{\circ} 46$ est de «supprimer le dispositif des ELCO».

32 http://cache.media.education.gouv.fr/file/DP_rentree_2017_NVB/96/8/2017_preparer_la_ rentree_F5_728968.pdf.

33 La Défenseure des Enfants, Actes du colloque Mineurs étrangers isolés, vers une harmonisation des pratiques pour l'intérêt supérieur des enfants, 20 juin 2008 [en ligne]. Disponible sur : http:// defenseurdesenfants.fr/pdf/Actes_MEI.pdf.

34 Cette catégorisation figure dans le rapport suivant : Étiemble Angelina et Zanna Omar, 2013, Des typologies pour faire connaissance avec les mineurs isolés étrangers et mieux les accompagner, Convention de recherche $n^{\circ}$ 212.01.09.14, "Actualiser et complexifier des motifs de départ du pays d'origine des mineurs isolés étrangers présents en France », Topik/Mission de Recherche Droit et Justice. 
travaux pratiques. Négligeant la théorie au profit du manuel, les instituteurs les destinent à devenir ouvriers. " ${ }^{35}$.

Enfin, un enfant "d'immigrés » peut être né sur le sol français et n'avoir jamais connu de pays étranger, ni d'autre langue que le français. La circulaire de $1978^{36}$ considère l'appréhension du public en fonction des parcours migratoires des parents et des difficultés en français, que l'enfant soit né ou non en France : les préconisations ne se limitent pas aux élèves arrivants mais concernent aussi les " enfants [qui] sont nés en France ou y résident depuis un temps suffisant pour parler notre langue " et qui sont repérés en difficultés. Les terminologies élargissent ou restreignent la prise en charge spécifique des élèves et les Français natifs peuvent être concernés pour peu qu'ils soient issus de l'immigration et avec une maîtrise insuffisante du français, ce qui sera remis en question, à travers les circulaires ultérieures.

\section{Des terminologies temporelle et géographique}

Où situer l'Autre sans l'exclure, ni le catégoriser à travers des stéréotypes? Plus neutre, l'expression "primo-arrivant " a traversé les années 90 jusqu'aux années $2000^{37}$. Toutefois, l'appellation perdrait sa popularité au début des années 2000 car le préfixe " primo » rappellerait " primo-infectieux » ${ }^{38}$, ce qui fait écho à une anecdote de l'enseignante Nadine Croguennec-Galland : "Quand il me croisait et qu'il était seul Pedro me disait bonjour, quand il était avec des camarades de sa classe, il m’ignorait. Il fuyait son passé de "primo-arrivant" comme une maladie, une sorte de "primo-infection" $"{ }^{39} .$.

La circulaire de 2002 désigne les "élèves nouvellement arrivés en France " 40 (ENAF), dénomination concurrencée par celle de " nouveaux arrivants " ${ }^{41}$, ce qui décrit un procès temporaire soit révolu (arrivé), soit en cours (arrivant). Depuis $2012^{42}$, la dernière lettre de l'acronyme ENAF a disparu et la mention

35 Dalila Kerchouche, Mon père ce harki, Seuil, 2003, p. 139.

36 Circulaire $\mathrm{n}^{\circ} 78-238$ du 25 juillet 1978 : Scolarisation des enfants immigrés.

37 Circulaire n ${ }^{\circ}$ 90-270 du 9 octobre 1990, Missions et Organisation des CEFISEM. Circulaire $\mathrm{DMP} / \mathrm{CI} 1 \mathrm{n}^{\circ} 99-315 \mathrm{du} 1^{\mathrm{er}}$ juin 1999, Circulaire relative à la mise en place du dispositif d'accueil des primo-arrivants. On retrouve l'expression aussi dans les textes régissant le Contrat d'Accueil et d'Intégration et différents supports édités par l'Éducation Nationale.

38 Nathalie Auger, Élèves nouvellement arrivés en France, Réalités et Perspectives pratiques en classe, Paris Éditions des archives contemporaines, 2011, p. 18.

39 Nadine Croguennec-Galland, La classe au bout du voyage, Le quotidien de jeunes migrants raconté par leur professeur, Paris, L'Harmattan, 2009, p. 56.

40 Circulaire $\mathrm{n}^{\circ}$ 2002-100 du 25 avril 2002, Organisation de la scolarité des élèves nouvellement arrivés en France sans maîtrise suffisante de la langue française ou des apprentissages.

41 Expression figurant, par exemple, dans la circulaire $n^{\circ}$ 77-447 du 22 novembre 1977, Enseignement de leur langue nationale aux élèves yougoslaves scolarisés dans l'enseignement élémentaire (serbo-croate, slovène, macédonien...).

42 Circulaire $n^{\circ}$ 2012-141 du 2 octobre 2012, Organisation de la scolarité des élèves allophones 
de la France ne figure plus, ce qui redéfinit une fois de plus les limites du public : par exemple, les élèves de la France d'outre-mer dont des besoins en français sont avérés peuvent alors relever des dispositifs lorsqu'ils arrivent en métropole, bien qu’ils aient effectué leur scolarité dans le système éducatif français.

Enfin, une autre expression "enfants venus d'ailleurs " se définit aussi en rapport avec cette arrivée inédite mais elle engage une décentration sur le départ et la situation antérieure non identifiés... ce que Cécile Goï élucidera dans un ouvrage du même nom ${ }^{43}$ ou encore Marie-Rose Moro avec une approche en éthnopsychanalyse ${ }^{44}$.

\section{Des terminologies liées aux compétences en français}

La place allouée se fonde donc sur des critères juridiques (" étranger »), sociaux (" enfant de travailleur immigré ») pour une durée indéterminée ou pour une durée déterminée encore que confuse ("arrivant ", "arrivé »). Pour toutes ces appellations qui permettent l'identification, la caractérisation commune est le degré de maîtrise en langue française. Dès la première circulaire, en $1970^{45}$, la dimension linguistique apparait avec la référence aux étrangers " non francophones ». C'est ce besoin implicite constaté par (et pour) les équipes éducatives qui a justifié une catégorisation spécifique au sein de la population scolaire pour organiser des dispositifs qui favorisent une acquisition rapide de la langue française et déjouent des risques d'échec scolaire. Cette appellation toutefois négative pointe la carence, le manque, la distance par rapport à une norme. Elle nous conduit à deux remarques si on la ramène à notre actualité. Tout d'abord, elle suscite le débat de la durée, qui persiste à travers les appellations : pendant combien de temps l'élève peut-il justifier d'une prise en charge spécifique ? Pour le moins, ici, cette phase de " non francophonie " est particulièrement temporaire : dès quelques semaines, des élèves se débrouillent en français, mais on mesure davantage les progrès à réaliser plutôt que les progrès réalisés. En second lieu, des élèves issus de pays francophones peuvent disposer de compétences satisfaisantes en production orale et être en situation proche de l'illettrisme, non pas pour des raisons intellectuelles, mais parfois en conséquence à leurs conditions d'apprentissage antérieures. C'est d'ailleurs ce qui avait conduit l'académie de Paris à mettre en place des dispositifs de Français Langue Écrite Renforcée (FLER).

nouvellement arrivés, Bulletin Officiel $\mathrm{n}^{\circ} 37$ du 11 octobre 2012.

43 Cécile Goï, Des élèves venus d'ailleurs, Orléans, CRDP d'Orléans, Les cahiers de Ville École Intégration, 2005, rééd. 2015.

44 Marie-Rose Moro, Enfants d'ici venus d'ailleurs, naître et grandir en France, La Découverte, Paris, 2002.

Op. cit. 
Les circulaires ultérieures vont plutôt référer à l'insuffisance de la maîtrise de la langue. C'est quelque peu forcé avec le texte de 1978 dans la mesure où la catégorisation ne se fait pas sur le seul critère de l'arrivée récente puisque des élèves nés en France d'un parent immigré peuvent être concernés, quand leurs compétences en français sont jugées insuffisantes. Si le critère n'est pas celui d'une arrivée en cours de scolarité, il concerne plutôt le degré de maîtrise du français en lien avec un bilinguisme avéré. De ce fait, la problématique de la maîtrise de la langue s'élargit au fil des années et il devient nécessaire de redéfinir les missions du service du rectorat en charge de ce dossier, les Centres régionaux de formation et d'information pour la scolarisation des enfants de migrants (CEFISEM), créés en $1976^{46}$ et restructurés en centres académiques pour la scolarisation des nouveaux arrivants et des enfants du voyage (CASNAV), par la circulaire de 2002. Cette année-là, les circulaires se centrent sur les nouveaux arrivants " sans maittrise suffisante de la langue française ou des apprentissages ", ce qui peut alors effectivement englober la catégorie des élèves issus de pays francophones. La circulaire de 2012 crée une décentration en imposant une autre épithète : "allophone ", qui fait valoir non pas le manque, mais une différence en contexte liée à la langue première et à l'arrivée récente. Toutefois, la compréhension de "allophone " par les enseignants est variable et certains de l'utiliser comme synonyme de "non francophone" (d'où des expressions comme "un élève est encore un peu allophone » ou " n'est plus du tout allophone " après quelques mois d'immersion). Le terme dont l'emploi était réservé à la phonologie en tant que variante combinatoire d'un phonème n'apparait que dans ce sens dans des dictionnaires de sciences du langage ${ }^{47}$ et est absent de dictionnaires des noms communs. Il est utilisé dans des ouvrages pédagogiques progressivement. Ainsi, Catherine Marcus, enseignante de français et formatrice en FLS, l'emploie pour évoquer l'élève dans ses relations aux langues en prise à la migration : "En situation de rupture, d'exil, l'élève allophone assiste à ce que A. Khatibi appelle "le silence de la langue maternelle" tout au moins pendant le temps de l'enseignement ${ }^{48}$ et plus loin, lorsqu'elle propose des pratiques pédagogiques désignées aujourd'hui en tant qu’approches plurielles, qui s'appuient les langues d'origine : "Notre propos n'est pas de survaloriser les langues des élèves allophones, mais de leur accorder la place qui est la leur

46 Circulaire n ${ }^{\circ} 76-387$ du 4 novembre 1976 portant sur la création des Centre régionaux de formation et d'information pour la scolarisation des enfants de migrants (CEFISEM).

47 Jean Dubois, Giacomo Mathée, Louis Guespin, Christiane Marcellesi, Jean-Baptiste Marcellesi et Jean-Pierre Mevel, 1994, Dictionnaire de linguistique et des sciences du langage, Larousse ; Oswald Ducrotet Tzvetan Todorov, Dictionnaire encyclopédique des sciences du langage, Points, éditions du Seuil, 1972 ; Robert Galisson et Daniel Coste(éds), Dictionnaire de didactique des langues, Hachette, 1976.

48 Catherine Marcus, op. cit., p. 52. 
dans la multiplicité des langues ${ }^{49}$. À cette période, le terme apparait dans les documents d'accompagnement des programmes de 1996 et 1999 : par exemple, pour les classes de $5^{\mathrm{e}}$ et de $4^{\mathrm{e}}$, il est fait mention que " le français langue seconde concerne les élèves allophones, souvent plurilingues ». Le terme " allophone " figure avec sa signification actuelle dans le dictionnaire dirigé par Jean-Pierre Cuq : "le terme bénéficie d'une acceptation plus large, employée pour catégoriser un public qui parle une langue "autre". Au Québec, par exemple, il désigne les élèves immigrés qui doivent suivre un enseignement spécifique [...] il souligne la différence linguistique, aux dépens de l'appartenance linguistique et culturelle. Aussi, dans la relation enseignant-apprenant, l'enseignant gagnera-t-il toujours à se demander qui est l'allophone de qui ${ }^{50}$. Toutefois, au Québec, le terme d'allophone est étendu aux élèves nés au Québec mais dont la langue maternelle n'est ni le français (voire ni l'anglais) ${ }^{51}$ tandis qu'il parait restreint - par l'usage - aux élèves récemment arrivés alors que le terme d'alloglotte émerge pour désigner les élèves nés en France ayant une langue maternelle autre que le français.

\section{La prise en compte de la variété des besoins éducatifs particuliers}

En revenant sur la circulaire de 2002, l'acception retenue prend aussi en compte l'insuffisance des " apprentissages scolaires » en relation avec le cursus antérieur ou le parcours individuel. Cette terminologie justifie la mise en place d'un enseignement spécifique, déjà développé précédemment, qui ne se limite pas à la langue française : par exemple, des collèges proposent des cours de mathématiques pour les élèves qui n’ont pas étudié la géométrie dans leur pays d'origine ou qui ont été peu scolarisés ou qui sont en difficultés. Quoique cette caractérisation n'ait pas été maintenue dans la circulaire de 2012, cela n'a pas modifié la conception des dispositifs proposant un enseignement spécifique dans plusieurs disciplines, même si la question de la langue demeure prépondérante. La dernière circulaire, de 2012, considère les élèves comme «à besoins éducatifs particuliers " (EBEP), ce qui est pressenti dans la circulaire de 2002, lors de l'université d'automne à Vichy ${ }^{52}$ ou encore en 2007 dans un encart ${ }^{53}$ qui recense les structures pédagogiques spécifiques.

49 Ibid., p. 53.

50 Jean-Pierre Cuq (éd.), Dictionnaire de didactique du français langue étrangère et langue seconde, Paris, CLE International, 2003, p. 17.

51 Françoise Armand, "Les élèves immigrants nouvellement arrivés et l'école québécoise ", in Santé, Société et Solidarité, $\mathrm{n}^{\circ}$ 1, Immigration et intégration, 2005.

52 Actes de l'université d'automne, Le système éducatif français et les élèves à besoins éducatifs particuliers, 30 octobre 2003, collection À Propos.

53 Encart 2007 : énumération des structures pédagogiques prévues aux décrets du 25 mai 1950 modifiés. 


\section{Des besoins éducatifs particuliers à l'évocation du handicap}

L'expression " élèves à besoins éducatifs particuliers " (EBEP) tisse des liens ténus avec le handicap. Pour mémoire, les élèves éprouveraient "des difficultés spécifiques dues à un handicap linguistique diffus et à une insertion partielle dans le milieu culturel français ", indique-t-on dans la circulaire de 1978 qui fait un constat commun aux élèves allophones natifs et arrivants. L'évocation du " handicap » est alors critiquée, la même année : " tout se passe comme si l'enfant étranger était effectivement un handicapé ${ }^{54}$. Signalons que les premiers dispositifs Dans le Livre Vert sur la scolarisation des enfants de migrants, au niveau européen, on rencontre 13 occurrences de " handicap " et ses dérivés, pour des handicaps linguistiques mais aussi socioéconomiques. Quel statut accorder aux aphasies des premiers temps?

La redéfinition du public comporte des enjeux : par exemple, elle laisse entrevoir la possibilité du tiers temps pour la passation d'examens ${ }^{55}$. Une proposition va dans ce sens, dans le rapport des Inspections Générales ${ }^{56}$ s'appuyant sur cette association : "les ENAF sont fortement "handicapés" lors des examens par leur manque de maîtrise de la langue française ". Les auteurs du rapport évoquent les aménagements opérés en Angleterre où des épreuves différentes sont proposées aux élèves.

Toutefois, du handicap linguistique au handicap mental, la frontière est parfois mince. Les orientations surreprésentées vers les sections spécialisées demeurent inquiétantes. En 1996, les sociologues Louis-André Vallet et JeanPaul Caille constatent que "l'enseignement spécial [avait] pu être détourné de sa vocation initiale pour scolariser des enfants que l'on ne savait scolariser ailleurs ». Une étude dirigée par Claire Schiff ${ }^{57}$ fait apparaitre des conclusions similaires et, en 2008, un rapport européen dresse le même constat, au niveau de l'Europe, cette fois de façon plus large, pour les enfants de migrants : ceux-ci sont surreprésentés dans les filières de l'enseignement spécialisé, réservé aux élèves présentant d'importantes difficultés scolaires liées par exemple à des handicaps ${ }^{58}$.

54 Claudine Gruwez, "Comment poser le problème ", in Patrick Besenval (éd.), Dans toutes nos classes, des enfants d'immigrés, Le Français aujourd'hui, revue trimestrielle, n 44, 1978.

55 Décret $n^{\circ}$ 2005-1617 du 21 décembre 2005, version consolidée au 18 octobre 2009, Décret relatif aux aménagements des examens et concours de l'enseignement scolaire et de l'enseignement supérieur pour les candidats présentant un handicap.

56 Catherine Klein et Joël Sallé, op. cit., p. 173.

57 Claire Schiff, op. cit.

58 Friedrich Heckmann (éd.), Education and migration, strategies for integrating migrant children in European schools and societies, NESSE report, European Commission, 2008, p. 8. 


\section{L'évolution des dispositifs}

Ces terminologies influencent celles des dispositifs. Les premières structures d'accueil s'appelaient classes d'adaptation ${ }^{59}$, ce qui n'est pas sans évoquer les structures homonymiques de l'époque, dédiées aux élèves en grandes difficultés scolaires (compétences inférieures au cycle 3) et, dans le rapport des Inspections générales de $2009^{60}$, puis confirmée avec la circulaire de 2012, s'impose l'UPE2A dont les deux premiers termes sont harmonisés avec l'UPI (Unité Pédagogique pour l'Intégration) réservée aux élèves présentant un handicap mental, visuel, auditif ou moteur. Ironie du sort, les UPI venaient d'être rebaptisées Ulis ${ }^{61}$, ce qui fit manquer le rendez-vous des acronymes harmonisés.

Entre temps, se sont développées diverses organisations de dispositifs ${ }^{62}$ pour lesquels il a fallu combiner moyens humains et moyens budgétaires en fonction du flux d'arrivées et en dépit du manque parfois avéré de formation des enseignants. Les contraintes structurelles mais aussi les représentations ont conduit à la création de dispositifs fermés à l'encontre des préconisations initiales dès la circulaire de $1973^{63}$ mais aussi des dispositifs ouverts tels que recommandé (les élèves sont obligatoirement rattachés à une classe ordinaire dans lesquelles ils suivent quelques heures) ou mixtes (les élèves quittent progressivement un dispositif fermé pendant quelques semaines, pour rejoindre progressivement une classe ordinaire). Actuellement, il est préconisé que l'élève soit inscrit dans une classe ordinaire du cursus régulier et éventuellement, dans une UPE2A ouverte et ce, pendant une année en moyenne (voire deux pour les élèves ayant des écarts de compétences en raison d'une scolarité antérieure discontinue), le critère de la durée prévalant sur celui des compétences pour éviter des rétentions abusives d'élèves en dispositif et pour des contraintes de nombre de places. Par ailleurs, la relation écolefamille est davantage prise en compte à travers des parcours formatifs destinés aux parents eux aussi allophones, de sorte qu'ils puissent mieux accompagner la scolarité de leurs enfants ${ }^{64}$.

59 On trouve l'évocation de "Classes d'adolescents non francophones ", qui correspondrait aussi à l'acronyme de CLAD, in Jean Clevy (éd.), Questions - réponses sur la scolarisation de enfants de travailleurs migrants, Les éditions de ESF, Collection SE (Science de l'éducation), 1976.

60 Catherine Klein et Joël Sallé, La scolarisation des élèves nouvellement arrivés en France, Paris, Ministère de l'Éducation Nationale, Ministère de l'Enseignement Supérieur, 2009.

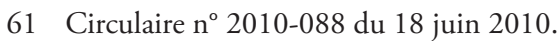

62 Catherine Mendonça Dias, op. cit., p. 116-134.

63 Circulaire $\mathrm{n}^{\circ} 73-383$ du 25 septembre 1973 : Scolarisation des enfants étrangers non francophones, arrivant en France entre 12 et 16 ans.

64 Circulaire n 2008-102 du 25 juillet 2008, Opération expérimentale, "Ouvrir l'École aux parents pour réussir l'intégration". 


\section{Des terminologies au regard des autres}

«Va te faire intégrer !», peut-on entendre dire ${ }^{65}$. Les regards et les mots des autres construisent la représentation identitaire du jeune qui s'y réfere. Étranger, enfant de travailleurs migrants, non francophone, allophone, arrivant puis arrivé, l'élève peut éventuellement être perçu plus étranger que les enfants de nationalité étrangère, davantage dépossédé de compétences langagières malgré son plurilinguisme, parfois déclassé socialement (c'est le cas d'enfant de journalistes, d'enseignants... dont les parents se retrouvent dans des emplois subalternes), en difficultés scolaires malgré son parcours brillant, etc. À lui de trouver sa place dans un nouvel établissement scolaire, en ajustant son propre regard sur lui-même et en acceptant celui porté par les autres, plus ou moins bienveillants. Les circulaires figent, un mot pèse, les phrases réduisent, le discours est pragmatique et instaure des cours spécifiques à un public qui se redéfinit sous le facteur commun du "manque » dans la biographie langagière. Maintenant que nous avons vu le regard porté par l'institution sur ces élèves, décentrons-nous pour envisager le regard que portent ces élèves sur cette institution, à travers leur rapport à la langue. Nous allons évoquer des difficultés autres que linguistiques qui tendent ou distendent la relation à la langue française.

\section{Les obstacles à l'apprentissage du français liés à la trajectoire migratoire}

Pour profiter pleinement de l'enseignement dispensé, l'élève doit adopter une posture d'apprenant volontaire et actif. Or, des facteurs individuels, parfois conjoncturels, peuvent entraver l'entrée dans la langue française. En effet, la trajectoire migratoire imprime une stabilité ou une instabilité du projet de séjour, du lieu de résidence et, de façon plus ou moins liée, draine et entraine des effets psychologiques, voire physiques (maux de tête, au ventre, grandes fatigues, prise ou perte de poids rapide...) qui en sont la cause ou la manifestation. Nous allons reprendre les cas de figure rencontrés pour comprendre la complexité de l'apprentissage du français en l'envisageant plus globalement, du pôle de l'enfant.

Pour ce faire, nous utilisons quatre procédés méthodologiques qui tiennent compte des compétences des élèves pour s'exprimer en français : nos analyses s'appuient sur des notes (les élèves notent leur scolarité, à plusieurs reprises dans l'année), des dessins (les élèves se représentent en France et dans leur pays d'origine), des entretiens semi-dirigés (avec captation ou non d'images) et des productions écrites (à travers un sujet d'imagination sur la rentrée scolaire).

65 Nathalie Auger, "Le rôle des représentations dans l'intégration scolaire des enfants allophones ", in Jean-Louis Chiss (éd.), Immigration, école et didactique du français, Paris, Didier, 2007, p. 215. 
Il a été intéressant de renouveler la même passation d'activités avec les élèves durant deux années scolaires pour observer leur ressenti, leur regard qui évolue sur le même sujet (à savoir, leur arrivée en France dans un nouveau cadre scolaire dont ils ne maîtrisaient pas la langue) et le lien avec l'apprentissage.

\section{Les incidences de la stabilité ou de l'instabilité du séjour et du domicile}

L'apprentissage scolaire et l'adaptation au nouvel environnement peuvent être conditionnés par le projet migratoire. Que celui-ci relève du regroupement familial, de la demande d'asile, de l'accueil chez un membre de la famille, de l'arrivée en tant que mineur non accompagné, de l'obtention d'un contrat de travail des parents, du retour de Français, de venue pour soins médicaux, de l'adoption ou encore d'échanges scolaires, le projet n'est pas anodin vis-àvis de l'inclusion scolaire. Il entraîne une stabilité ou une instabilité du séjour et du domicile, ce qui complexifie l'intégration scolaire : comment trouver sa place quand on va et vient, on vient et revient en France au gré des contrats de ses parents ou lorsqu'après quelques semaines, au cours de la procédure de la demande d'asile, on est obligé de changer d'hôtel, puis de foyer... ? Miguel est arrivé au collège un peu comme un touriste. Au bout de quelques mois, il s'est tourné vers le travail et s'est investi, commençant à construire son projet professionnel, hésitant sur le choix de son futur lycée... puis il est reparti un mois au Mexique, et puis il est revenu, il a rattrapé le travail, s'est concentré et un jour, il a appris qu'il quittait définitivement la France. Le lendemain. Voici sa lettre d'au revoir à l'enseignante : «Bonjour madame. Je vous remercie pour toute votre aide. Bon... Le première jour que j'arrive dans ce collège je suis enchanté de language français, j'adore la politesse. Et je vais partir à Mexique avec beaucoup de connaissance française. [...] C'est très difficile pour moi, c'est la chose plus difficile jamais faite dans ma vie. Mais je dits tout change pour le mieux ». Le retour est parfois imprévu, soudain, déstabilisant, comme le fut parfois l'arrivée : dans certaines familles, on ne dit pas forcément les choses aux enfants, quelques uns pensent venir une semaine en vacances chez une tante et ne reviendront pas au pays avant leur majorité.

En ce qui concerne le rapport entre le domicile et l'établissement, remarquons que le fonctionnement de l'UPE2A peut instaurer une séparation entre l'environnement scolaire et le quartier : en effet, le dispositif regroupe parfois des élèves qui relèvent de différents secteurs et prennent des transports avant de parvenir dans leur collège (cette modalité ne s'applique pas au niveau de l'école, l'enfant étant trop jeune). Deux conséquences : le quartier n'est pas connu via le milieu scolaire et, quand la période d'inscription en UPE2A arrive à terme au bout d'une année, il faut regagner un collège de secteur inconnu où reconstruire de nouveaux repères et un réseau d'amis, tout en revivant, autrement, l'expérience d'être nouveau. Pour Esteban, collégien 
de 12 ans, lorsqu'il se représente au Mexique, on le voit dans son école, un bâtiment avec des élèves et lorsqu'il se représente en France, il figure dans un bus : la scolarité pour lui est un voyage, celui qui l'a amené du Mexique aux États-Unis, puis des États-Unis en France et puis, sur Bordeaux, dans ce bus qui rythme ses journées entre le collège et son domicile.

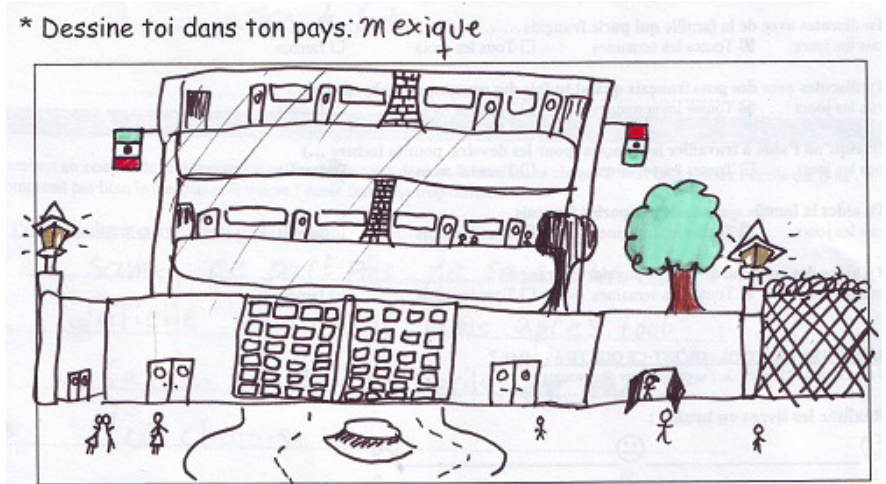

Document 1 : Esteban se représente au Mexique (test 2).

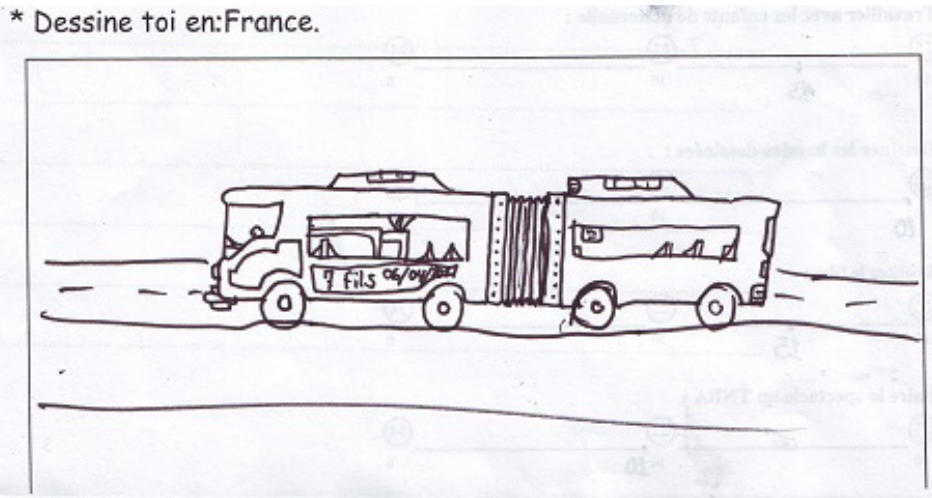

Document 2 : Esteban se représente en France (test 2).

\section{Phénomènes psychologiques et physiques}

Cette instabilité est relativement problématique en fonction de la personnalité, du parcours antérieur et des perspectives migratoires, en raison desquels l'enfant peut avoir été fragilisé. Ainsi, si l'élève doit s'adapter "à une autre culture d'apprentissage, à d'autres rituels scolaires, tout en apprenant la langue de scolarisation au plus vite ${ }^{66}$ dans un cadre à la violence symbolique (au sens

66 Fabienne Leconte, Clara Mortamet, "Les représentations du plurilinguisme d'adolescents scolarisés en classe d'accueil ", dans Fabienne Leconte, Sophie Babault, Construction de compétences plurielles en situation de contacts de langues et de cultures, Rouen, Glottopol $\mathrm{n}^{\circ}$ 6, juillet 2006, p. 22-57. 
de Bourdieu) accrue par l'éloignement culturel, on ne peut ignorer plusieurs phénomènes psychologiques qui freinent parfois le processus comme l'évoque Cécile Goï ${ }^{67}$ : "À l'école, beaucoup se confrontent à l'altérité, à la différence et pour eux, il est très possible d'éprouver une certaine peur d'apprendre " pour des motifs complexes tels que la honte, le conflit de loyauté, l'obligation au secret, le tabou, le mutisme, s'autoriser à réussir, etc. En effet, l'élève peut ne pas réussir à s'impliquer dans l'apprentissage du français. Rappelons que ce n'est jamais l'enfant qui a choisi de migrer mais ses responsables. Ce que Gilles Verbunt remarquait au sujet des adultes peut s'appliquer aux jeunes : "Ce n'est pas parce qu'un étranger se trouve en France qu'il ressent le besoin d'apprendre la langue française ${ }^{68}$. Le départ du pays initie parfois une période de grande tristesse comme pour Catarina, jeune Portugaise qui se représente le visage couvert de pleurs et qui, pour reprendre l'expression portugaise intraduisible qui rythme le fado, éprouvera la saudade.

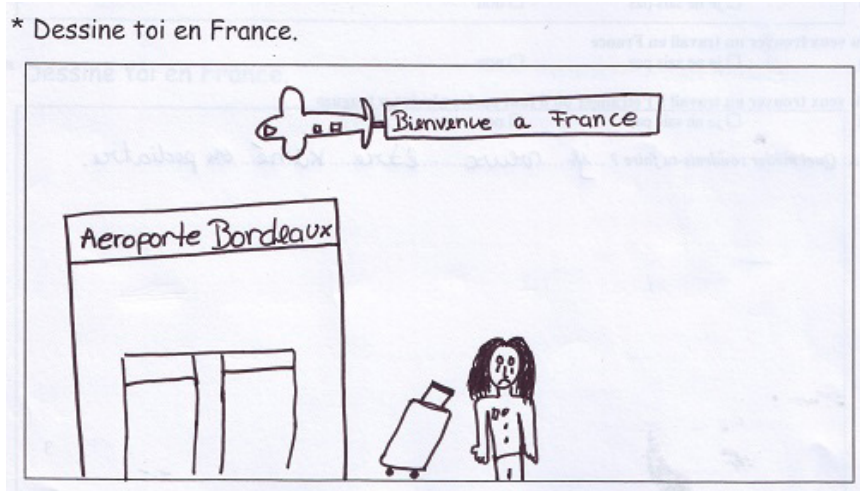

Document 3 : Catarina en France.

Parmi les phénomènes identifiés, relevons celui de " conflit de loyauté » cité par Cécile Goï ${ }^{69}$. Ce concept, issu des situations de divorce où l'enfant se sent obligé de prendre parti pour son père ou sa mère, est transférable à l'enfant qui se sent obligé de choisir entre sa culture d'origine et la culture d'accueil. Est-ce que parler la langue française n'est pas une trahison par rapport aux siens demeurés au pays ? Le jeune Esteban, dont nous avons présenté des dessins, a laissé son père au Mexique et lorsqu'il a réalisé la première fois les dessins dans lesquels il se représente, il n'avait pas choisi l'école mais des symboles sociaux, traités avec l'ironie «Bienvenido! ». Au Mexique, une arme et en France, une moto.

67 Cécile Goï, op. cit., p. 65.

68 Gilles Verbunt, Apprendre et enseigner le français en France, Paris, L'Harmattan, 2006, p. 14.

69 Cécile Goï, op. cit. 


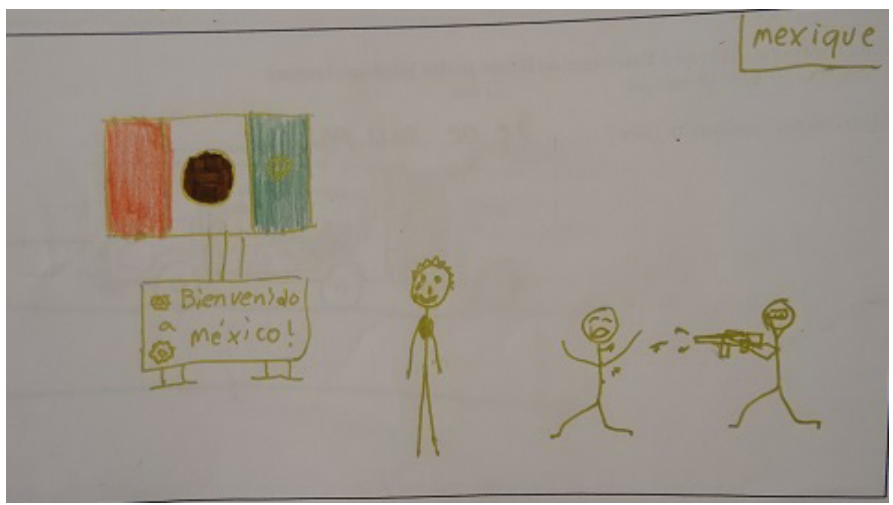

Document 4 : Esteban se représente au Mexique (test 1).

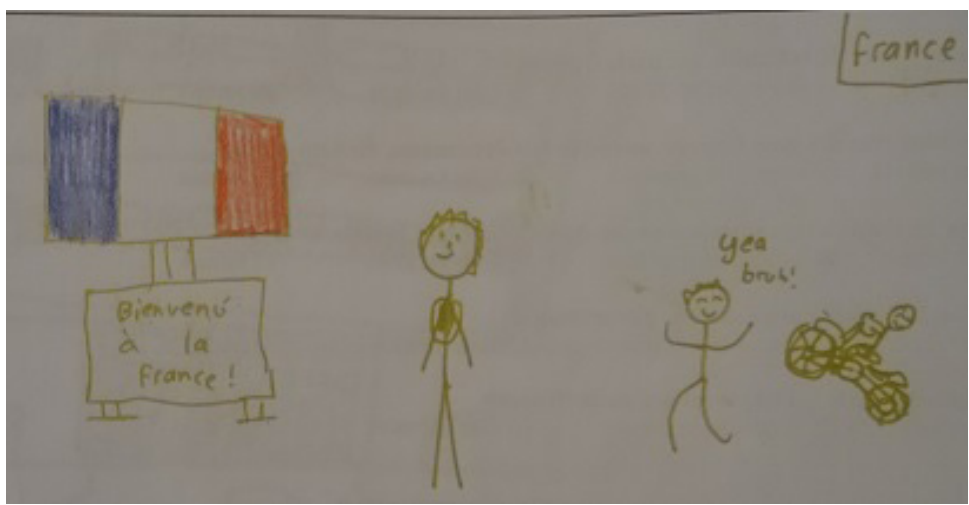

Document 5 : Esteban se représente en France (test 1).

En ce qui concerne la discordance entre culture d'origine et culture d'accueil, elle peut se manifester à travers un mutisme électif des enfants en milieu extra-familial, ce que constatent des éthnopsychologues comme Isam Idris ${ }^{70}$ : des jeunes s'expriment librement en milieu familial, mais se taisent à l'école. Gilles Verbunt ${ }^{71}$ rapporte quant à lui une situation où deux enfants turcs ne s'expriment pas, ni en français, ni en turc : le père, bien inséré, leur impose de parler le français tandis que la mère, désireuse de repartir, leur impose de parler en turc... Et d'observer qu' « il se peut que dans une famille, il y ait des stratégies contradictoires relatives au retour au pays" (ibid.). Autre silence, celui qui est lié au secret : pour des enfants de demandeurs d'asile ou des mineurs isolés, l'éducation au silence les a rendus silencieux. La parole risque de trahir, dénoncer, menacer, humilier.

70 Isam Idris, «Du culturel au thérapeutique : la vulnérabilité spécifique des enfants de migrants comme outils de réussite ", Les Conférences du CASNAV, Académie de Paris, t. 3, recueil des actes 2004 à 2005, 2005, p. 88.

71 Gilles Verbunt, op. cit., p. 50. 
D'autres enfants vont être silencieux, à la maison et à l'école, passifs et comme dépressifs, sans que des événements extérieurs autre que l'installation en France aient troublé la cellule familiale. Nous pouvons évoquer le cas d'une jeune fille tamoule, Piraï, originaire du Sri Lanka. Près de deux ans après son arrivée, elle s'est dessinée dans un même cadre inchangé, pour le Sri Lanka et la France. Notons que Piraï habite en milieu urbain en France. L'année suivante quand elle se représente en France, elle ne figure plus sur le dessin : ne reste que le paysage et sa ligne d'horizon.

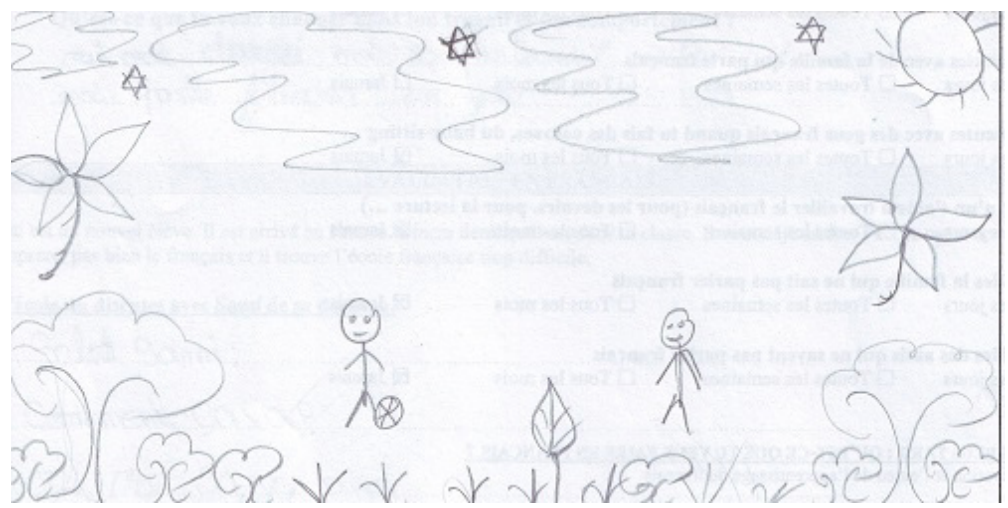

Document 6 : Piraï se représente au Sri Lanka.

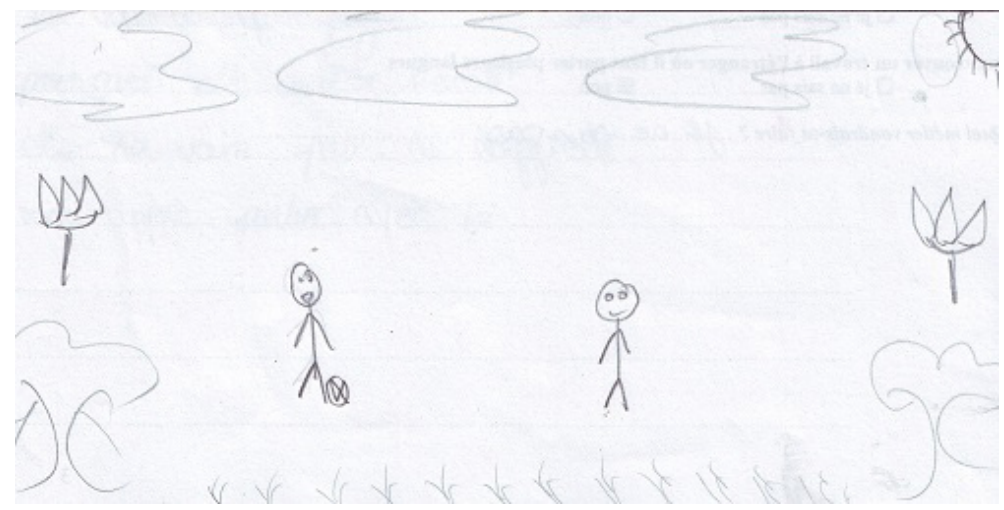

Document 7 : Piraï se représente en France.

Cette jeune fille n'est pas entrée dans la langue française : au bout de deux ans, le niveau A1 n'est pas atteint à l'oral. Elle demeure très passive, sans interaction avec ses pairs. Lors de sa troisième année, elle parvient au début du niveau A2. Son cas n'est pas isolé.

Dans cette présentation des extrêmes, quand ce n'est pas la langue française qui est rejetée, c'est la langue maternelle qui est écartée, refoulée ou oubliée (cas du bilinguisme soustractif) : cette attitude est observable par exemple chez des enfants adoptés comme l'auteur-illustrateur Sik-Jun Jung 
qui, dans une bande dessinée autobiographique, se représente enfant criant "Chuis plus coréen, chuis plus coréen ! " ${ }^{72}$.

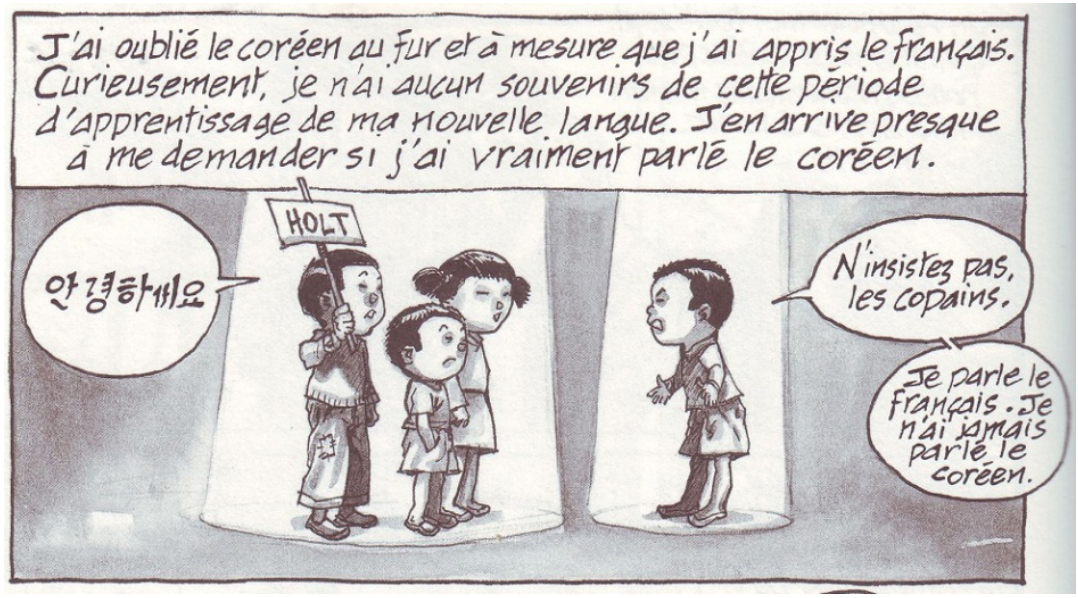

Document 8 : Extrait de Couleur de peau : miel.

Toutefois, s'il est vrai que paradoxalement l'objectif de l'apprentissage de la langue seconde, spécifiquement en contexte métropolitain et dans la tradition française, est bien souvent de l'amener à un statut de langue première, quitte à tendre vers le monolinguisme ${ }^{73}$, on peut alors poser la question du devenir plurilingue. Conscients du difficile équilibre à trouver, les acteurs de l'Éducation Nationale se sont interrogés sur la distinction entre l'intégration et l'assimilation : "Par le français, l'élève doit pouvoir s'intégrer dans une nouvelle communauté de vie et de travail, sans pour autant renoncer à être lui-même ni perdre ses propres repères ${ }^{74}$. Perte de repère, enrichissement culturel... l'" entre-deux " est cet espace entre la sphère d'origine et le nouveau contexte parfois chargé d'insécurité, où l'on est tout à la fois le même et différent. Ce qui est certain sur le terrain, c'est qu'aucun profil d'élèves, aucun récit de vie ne peuvent laisser présager du rapport ultérieur à la langue seconde et d'ailleurs, en ce qui concerne les migrations traumatisantes pour lesquelles on pourrait supposer des phases de silence, de deuil linguistique ou de rejet, le concept de résilience développé par Boris Cyrulnik ${ }^{75}$ prend tout son sens et c'est parfois avec de grands éclats de rire que l'élève s'accapare de sa nouvelle langue.

Par exemple, nous retrouvons plusieurs dessins où l'élève se représente dans un cadre identique, avec une posture, une expression et une activité identiques. Cette représentation n'est pas forcément symbolique du même

72 Jun Jung-Sik Jun, Couleur de peau : miel, Quadrants, 2007, p. 50.

73 Jean-Pierre Cuq, 1991, op. cit., p. 140.

74 Denis Bertrand, Alain Viala et Gérard Vigner, op. cit., p. 5.

75 Boris Cyrulnik, Un merveilleux malheur, éditions Jacob, 1999. 
processus : pour Piraï, c'est effectivement la fixation dans le temps et le deuil du pays d'origine ne s'est pas réalisé, en même temps qu'elle n'acquière la langue française qu'à force d'immersion. Le quartier, les amis, la famille manquent et elle attend un retour qui ne surviendra pas. Ismail, un adolescent albanais, est arrivé dans le cadre de la demande d'asile avec les siens dans des conditions difficiles sur le plan psychologique, renforcées par les conditions de séjour, avec des périodes dans la rue. En quelques mois, alors qu'il est non francophone à son arrivée, il arrive en tête de classe ordinaire, grâce aux solides compétences qu'il avait développées. Il ne laisse rien apparaître des obstacles et difficultés qu'il rencontre.

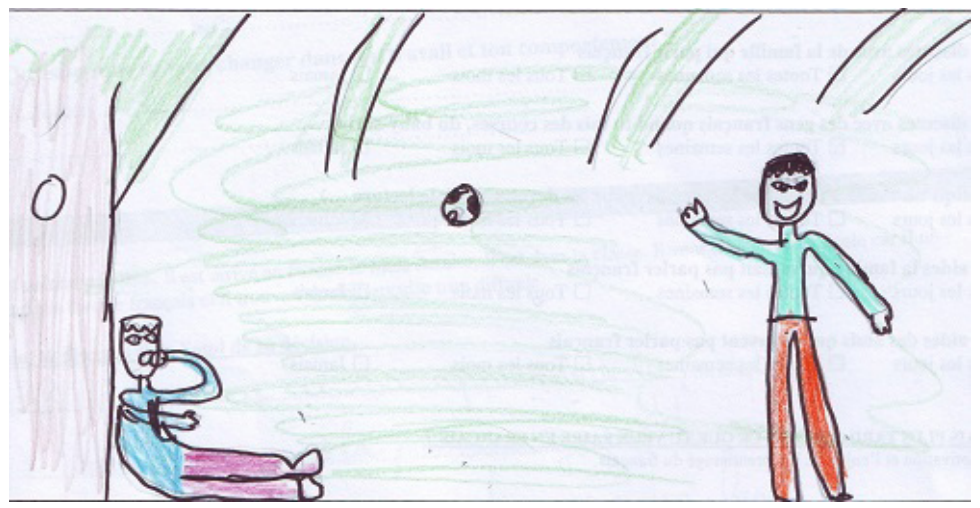

Document 9 : Alban se représente en Albanie.

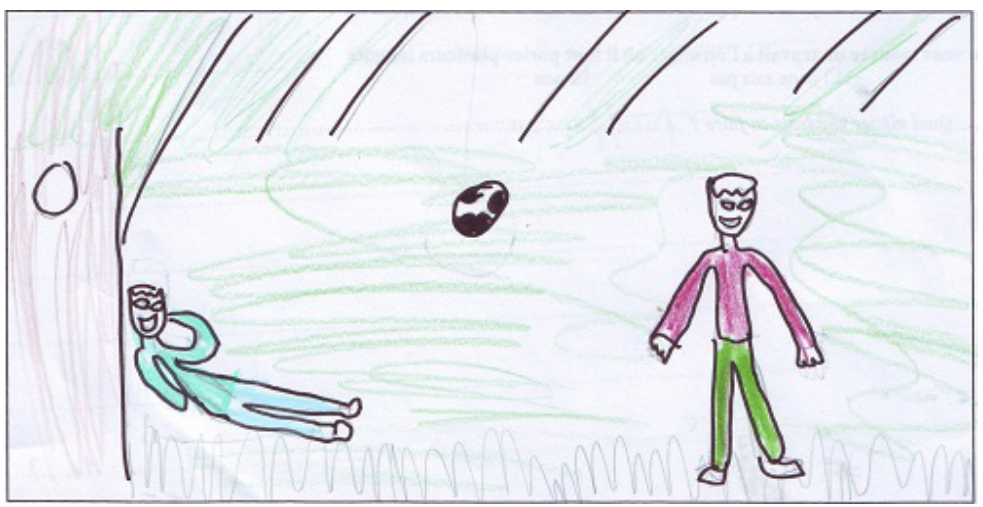

Document 10 : Alban se représente en France.

Ainsi, l'entrée dans la langue française peut être spectaculaire comme elle peut être différée de plusieurs semaines, de plusieurs mois, sans que cela ne soit forcément conscient de la part de l'enfant, ni forcément compris par l'enseignant qui juge à l'aune des compétences scolaires attendues. Difficile alors de comparer les élèves entre eux comme s'il existait un rythme unique d'apprentissage. Pourtant, la prise en charge spécifique ne tient pas en compte des compétences individuelles atteintes. 


\section{L'apprentissage d'une langue seconde et le silence forcé}

Quels qu'en soient les motifs, la période d'attente avant de communiquer en français coïncide avec une obligation de silence et de monologues intérieurs suppléant l'incapacité à s'exprimer de façon abstraite. Ci-dessous, on peut voir dans le dessin de Carlos, adolescent portugais, sa représentation dans le groupe d'amis, jouant en foot en bas des immeubles, tandis qu'on l'entrevoit à la fenêtre, au $3^{\mathrm{e}}$ étage d'un immeuble, enfermé dans sa chambre en France, occupé à jouer aux jeux en réseau. Les relations virtuelles compensent les relations amicales de l'adolescence.

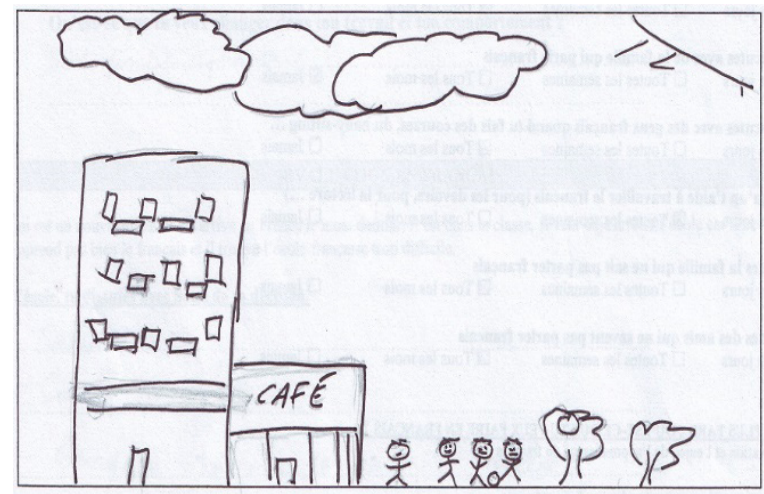

Document 11 : Carlos se représente au Portugal.

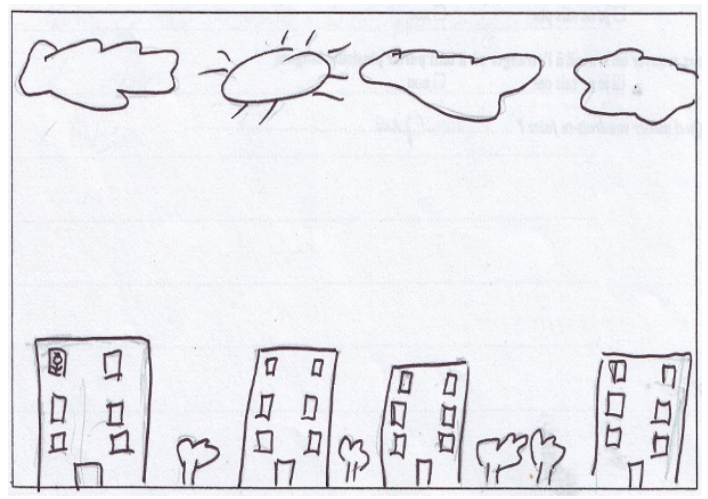

Document 12 : Carlos se représente en France.

Quelques mois ou années après leur arrivée, les élèves peuvent mettre des mots sur des émotions distanciées. Guillemette, collégienne arrivée en France suite à une adoption, se souvient, un peu plus de deux ans après son arrivée en France : «Moi, quand la première fois je suis arrivée ici, je trouvais l'école française très nulle. Je n'avais ni amis ni famille. Je me sentais toute seule, comme si dans une salle j'étais toute seule, qu'il faisait très noir et comme si j'appelle, 
je crie, je hurle... mais personne ne m'entend... $»^{76}$. Un espace révélateur à observer est la cour de récréation après le temps du réfectoire : c'est alors qu'on voit se répartir les groupes d'amis et l'isolement de s'imposer lorsque le groupe n'est plus régi par la cohésion de l'enseignant. Remarquons aussi que lorsque la communication ne passe pas par les mots, le contact physique et le nonverbal peuvent compenser, sous différentes formes, les moyens d'expression, plus ou moins bien accueillis par les adultes qui les jugent.

\section{Le rapport aux autres à partir du dispositif}

Pour certains élèves, le dispositif linguistique a alors un double enjeu, à la fois dans la construction des compétences en français mais aussi l'intégration dans un groupe de pairs qui lui reconnaissent une histoire commune. Il est alors complémentaire de l'inclusion. Une collégienne Monique, résidant en France depuis près de 3 ans, explique : "Dans notre établissement et un peu partout, ils ont installé des cours des fle pour des personnes qui ont de difficultés [...] dans les classes des fle, tu feras connaissance de beaucoup d'autres personnes comme toi. » : ce n'est pas tant l'apprentissage du français qui marque les souvenirs de cette collégienne, mais le partage d'expériences qui rompt avec la singularité parfois gênante de son histoire. Il arrive que le rattachement dans la classe ordinaire soit mal vécu.

16 élèves d'UPE2A notent...

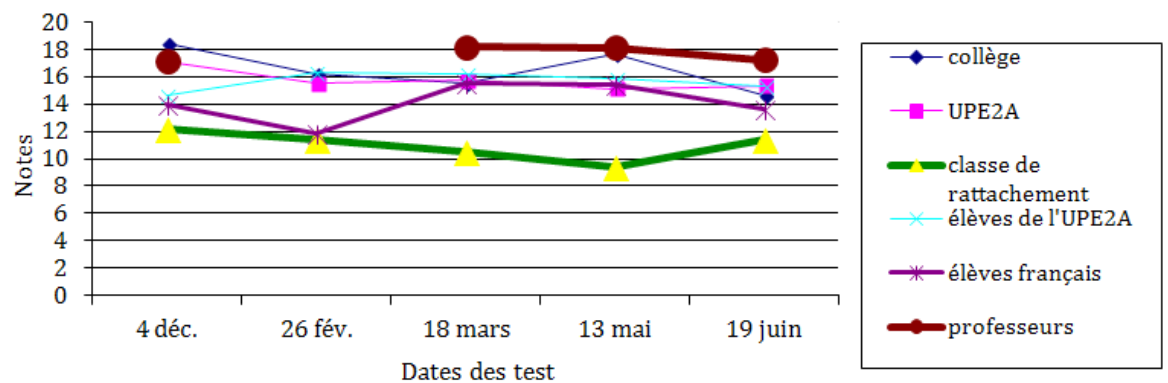

Document 13 : Les élèves notent leur collège.

En effet, des collégiens très peu francophones ont apporté leur ressenti et leur jugement sur leur environnement scolaire à travers des notes, au fil de l'année. Il est apparu qu'ils avaient une image très positive de leurs enseignants mais un regard plutôt négatif sur leur intégration en classe ordinaire : au mois de mai, lorsque les élèves notent leur classe de rattachement, celle-ci n'obtient même pas la moyenne. Par exemple, Sali, élève d'origine bulgare, attribue un 20 quelques jours après son arrivée, puis le restant de l'année, 
note sa classe de rattachement entre 0 et 5 . De rares élèves notent très favorablement leur classe ordinaire : Souleymane, lusophone scolarisé en inclusion avant de bénéficier quelques heures en dispositif, demeure très positif vis-à-vis de sa classe ordinaire où il s'est parfaitement bien intégré d'autant qu'il participe à la section foot. Aujourd'hui, l'école fait le pari de l'inclusion. Toutefois, celle-ci s'organise et se prépare notamment à travers la formation des enseignants sensibilisés aux problématiques de l'éducation interculturelle. Que ce soit avec l'appui d'un dispositif ou partiellement ou directement en inclusion, la qualité sociale et le bien-être facilite cet apprentissage de la langue, auquel cas plusieurs leviers peuvent être envisagés, notamment à travers la relation école-famille et la formation continue des enseignants. Ces besoins éducatifs particuliers ne peuvent être envisagés qu'avec du temps et dans le temps, ce que vont faire apparaittre des rythmes d'apprentissage que nous présentons ci-après.

\section{L'acquisition de compétences en français pour une intégration réussie}

Les élèves, marginalisés ou distingués en raison de leur apprentissage récent de la langue française, vont entrer progressivement dans la langue française, plus ou moins rapidement, plus ou moins aisément, plus ou moins volontairement, en interaction avec les locuteurs natifs ou en utilisant le français comme langue véhiculaire dans le groupe d'élèves d'allophones. Nous allons mettre maintenant en évidence des rythmes d'apprentissage ${ }^{77}$.

\section{Présentation de l'échantillon}

Nous nous appuyons sur une cohorte au départ constituée de 190 collégiens, arrivés entre septembre 2008 et juin 2009 dans une même académie. Il s'agit de 97 garçons et 93 filles, âgés de 10 à 17 ans, originaires de 45 pays, les plus représentés étant ici la République Démocratique du Congo, la Turquie et le $\mathrm{Maroc}^{78}$. Les raisons de leur arrivée sont diverses : près des trois quarts, de nationalité française ou non, viennent rejoindre leurs parents, voire un membre de famille, tandis que les autres arrivent dans le cadre de demande d'asile, de contrats de travail des parents, d'adoption, d'échanges scolaires ou des isolés se retrouvent pris en charge par l'Aide Sociale à l'Enfance.

Locuteurs de 48 langues, $70 \%$ étaient scolarisés antérieurement avec leur langue première et $30 \%$ avaient commencé leur scolarité avec une langue seconde. Tous connaissaient l'alphabet latin, utilisé pour leur langue de scolarisation ou leur langue vivante (généralement l'anglais). Ils avaient une

77 Catherine Mendonça Dias, op. cit.

78 Sur le plan national, il s'agissait alors du Portugal, de la Turquie et de la Chine, au collège (cf. Catherine Klein et Joël Sallé, op. cit., p. 18). 
connaissance variable du français : moins de la moitié n'en avait aucune connaissance en arrivant tandis que les autres se débrouillaient plus ou moins, avec un niveau inférieur au B1 d'après le CECRL (Cadre Européen Commun de Référence pour les Langues) ${ }^{79}$, qu'ils aient eu le français comme langue de scolarisation ou comme langue vivante étudiée à l'école (dans ce dernier cas, le niveau était plutôt le A1).

Près des trois quarts ont suivi des cours de français dans un dispositif spécifique tandis que les autres étaient soit éloignés géographiquement, soit avec un niveau en français similaire à des élèves francophones natifs. Dans l'académie de l'étude, l'inscription dans un dispositif n'était pas limitée dans le temps, alors les élèves ont pu suivre les cours jusqu'à ce qu'ils n'en aient plus besoin ou jusqu'à leur orientation en lycée pour les plus âgés. Ainsi, quand nous avons retrouvé 114 élèves ${ }^{80}$, près de trois ans après leur arrivée, 67 élèves d'entre eux bénéficiaient encore de cours dans un dispositif, à raison de quelques heures.

\section{Les outils pour évaluer des performances linguistiques}

Pour estimer le niveau linguistique atteint au cours des trois premières années, nous nous appuyons sur trois indicateurs. Le premier concerne les compétences initiales, évaluées en langue de scolarisation d'origine, à l'arrivée des élèves en France par un formateur du CASNAV. Cette évaluation initiale permet de repérer quelques compétences en compréhension écrite, ainsi qu'en mathématiques comme l'indique Mickaël Rigolot, formateur : " il s'agit avant tout de conduire une observation précise du patrimoine scolaire de l'élève et d'apprécier sa proximité avec les propositions et les attentes de l'école française, informations utiles aux professeurs ainsi qu'aux personnels de direction et de vie scolaire $"{ }^{81}$. Cette identification est d'autant plus importante quand il s'agit d'élèves non ou peu scolarisés antérieurement, parfois repérés tardivement ${ }^{82}$. Les résultats permettent d'apporter des données quant aux compétences scolaires à l'arrivée.

79 Conseil de l'Europe, Division des politiques linguistiques, 2000, Cadre européen commun de référence pour les langues : apprendre, enseigner, évaluer, Strasbourg.

80 Concernant les élèves " non retrouvés ", 4 étaient déscolarisés, 7 étaient retournés dans leur pays, 22 avaient déménagé hors de l'académie et 43 élèves avaient changé d'établissement mais n'ont pas été retrouvés dans l'établissement d'accueil indiqué, principalement en lycée. Cette mobilité en fait un public difficile à suivre.

81 Mickaël Rigolot, "Construire la connaissance autour de l'élève nouvellement arrivé en France ", in Klein Catherine (éd.), Le français comme langue de scolarisation. Accompagner, enseigner, évaluer, se former, Collection Cap sur le français de la scolarisation, Scéren, CNDPCRDP, 2012, p. 58.

82 Catherine Mendonça Dias, «Les progressions linguistiques et scolaires par les collégiens nouvellement arrivés, non ou peu scolarisés antérieurement ", Revue Recherches en didactique des langues et des cultures (RDLC) : les cahiers de l'Acedle, vol. 10, $\mathrm{n}^{\circ}$ 1, octobre 2013, p. 159-175. 
Le second indicateur s'appuie sur les résultats au DELF scolaire (Diplôme d'Études de Langue Française), examen de français facultatif, passé par 106 élèves de la cohorte, au cours de leurs trois premières années en France. Il permet d'évaluer les compétences communicatives en compréhension et production écrites et orales.

Le troisième indicateur correspond à un test linguistique que nous avons créé et soumis à 80 élèves de la cohorte, lors de leur troisième année en France, en 2011. Encore collégiens ou devenus lycéens, de la $5^{\mathrm{e}}$ à la $2^{\text {nde }}$, ils étaient répartis dans 28 établissements, la plupart en zones RAR (Réseau Ambition Réussite) ou RRS (Réseau de Réussite Scolaire). Nous leur avons fait passer un test linguistique d'une durée de 50 minutes, composé d'un exercice à closure, d'une compréhension de texte (extrait de PISA) et d'une rédaction. Afin de situer leur niveau, les mêmes exercices ont été proposés à 77 élèves natifs, scolarisés en classe type.

\section{Les progrès en français}

En observant les résultats au DELF, lors de la $2^{\mathrm{e}}$ année, $77 \%$ des élèves inscrits (soit 55 candidats) réussissent le niveau $\mathrm{A} 2$, voire $\mathrm{B} 1$, alors que les 16 autres candidats n'obtiennent pas la moyenne ou sont au niveau A1. Toutefois, ces pourcentages ne font pas état des élèves non inscrits car leur niveau était insuffisant, élèves que nous retrouvons parfois l'année 3 , inscrits au DELF A1 ou A2 (12 cas dans notre cohorte).

Observons maintenant les résultats de notre troisième indicateur : le test linguistique, soumis lors de l'année 3. Plus de $20 \%$ des élèves de la cohorte, qui ne connaissaient généralement pas le français à leur arrivée en France, obtiennent une note inférieure à 5 sur 20 (ce qui n'est pas arrivé avec les collégiens natifs qui ont réalisé les mêmes exercices). Leurs copies se caractérisent par un encodage phonétique de mots et l'incapacité à exprimer sa pensée en français, à l'écrit. Ces élèves se répartissent en trois catégories : les peu scolarisés antérieurement, ceux en échec scolaire dans leur pays d'origine et ceux qui utilisent très peu le français oral hors de l'école, n'adhérant pas toujours au projet migratoire, d'après leurs enseignants.

En revanche, l'écart n'est pas significatif entre les notes insuffisantes, entre 5 et 10 . De même, l'écart parmi les meilleures copies, au-dessus de 15, est plus réduit. En moins de trois années de scolarisation, un groupe d'élèves parvient à atteindre un niveau comparable aux natifs et parmi les plus brillantes copies, tout profil confondu, on compte 3 élèves qui ne connaissaient pas un mot de français à leur arrivée. L'hétérogénéité caractérise leur profil sur le plan de la langue (bulgare, chinois, edo), de l'âge (12 et 15 ans), du projet migratoire (demande d'asile, regroupement familial) et du niveau social. Seul point commun : de bons résultats lors du test initial en langue d'origine. On 
retrouve cette corrélation entre la progression linguistique et les compétences scolaires initiales avec les élèves ayant eu français en langue vivante, scolarisés régulièrement : ceux qui avaient de bonnes compétences en compréhension écrite et en mathématiques dans leur langue d'origine obtiennent de meilleurs résultats en français. Ils rattrapent un niveau proche de celui des élèves issus des pays francophones.

\section{La relation entre les progressions linguistiques et les parcours scolaires}

Gérard Vigner estime que le brevet nécessite d'avoir un niveau compris entre le B2 et le C1, d'après le CECRL (Vigner, 2008 : 39). Les niveaux atteints par la cohorte sont moindres mais le niveau B1 écrit est toutefois suffisant et représentatif des élèves obtenant le brevet ; à l'inverse, ceux qui quittent le collège sans brevet ont un niveau inférieur au B1, sauf exceptions ${ }^{83}$. Ce niveau est avéré par les résultats au DELF et/ou au test linguistique, sachant que le niveau B1 était nécessaire pour obtenir la moyenne au test linguistique.

Les élèves qui réussissent le brevet ne sont pas seulement ceux qui avaient le français comme langue de scolarisation dans leur pays d'origine, loin s'en faut car les élèves qui avaient étudié le français en langue vivante réussissent en majorité le brevet, mieux que les élèves au départ non francophones à leur arrivée, dont la maîtrise du français est encore insuffisante pour cet examen, que ce soit lors de leur $2^{\mathrm{e}}$ année en France ou leur $3^{\mathrm{e}}$ année.

\begin{tabular}{|c|c|c|c|c|c|c|c|}
\hline & \multirow{2}{*}{ 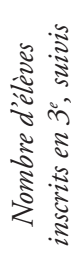 } & \multicolumn{2}{|c|}{$\begin{array}{c}\text { Elèves qui avaient le } \\
\text { français comme langue } \\
\text { de scolarisation dans leur } \\
\text { pays d'origine }\end{array}$} & \multicolumn{2}{|c|}{$\begin{array}{l}\text { Elèves qui avaient } \\
\text { étudié le français en } \\
\text { tant que langue vivante }\end{array}$} & \multicolumn{2}{|c|}{$\begin{array}{c}\text { Elèves qui n’avaient } \\
\text { aucune compétence en } \\
\text { français à leur arrivée } \\
\text { en France }\end{array}$} \\
\hline & & ADMIS & $\begin{array}{l}\text { NON ADMIS } \\
\text { OU NON } \\
\text { INSCRIT }\end{array}$ & ADMIS & $\begin{array}{l}\text { NON ADMIS } \\
\text { OU NON } \\
\text { INSCRITS }\end{array}$ & ADMIS & $\begin{array}{l}\text { NON ADMIS } \\
\text { OU NON } \\
\text { INSCRITS }\end{array}$ \\
\hline AN 2 & 37 & 6 & 5 & 5 & 6 & 4 & 11 \\
\hline AN 3 & 40 & 7 & 6 & 8 & 2 & 5 & 12 \\
\hline
\end{tabular}

Document 14 : L'obtention du brevet par 35 élèves de la cohorte, lors de leur $2^{\mathrm{e}}$ ou $3^{\mathrm{e}}$ année en France.

Cependant, si l'obtention du brevet est liée au niveau atteint en langue française, elle est aussi corrélée aux compétences scolaires initiales. Plus l'élève avait de bons résultats à l'évaluation initiale (en langue scolaire d'origine), meilleures semblent ses chances d'obtenir le brevet. De plus, les élèves admis étaient plus nombreux à avoir une connaissance de la langue anglaise, atout

83 Des élèves admis au DELF B1 et ajournés au brevet n'avaient été, en fait, " pas inscrits " au brevet par leur établissement, de peur de compromettre le passage en filière générale. 
précieux pour la validation du brevet. Observons enfin que, dans cet échantillon, les filles réussissent mieux que les garçons, ce qui ne déroge pas aux statistiques nationales.

Cette réussite s'accompagne d'une orientation en filière générale ou en bac professionnel par choix personnel. Les autres élèves, qui sortent du collège sans le brevet, s'orientent en filière professionnelle, généralement en Certificat d'Aptitude Professionnelle (CAP), ou sont pris en charge par un dispositif de la Mission Général d'Insertion (MGI), aujourd'hui rebaptisée Mission de Lutte contre le Décrochage Scolaire (MLDS). On relève quelques cas de déscolarisation liée au rapport à la culture scolaire plutôt que résultant de difficultés linguistiques. En effet, ces élèves - des garçons (sauf une jeune fille devenue mère et une autre, malade) - se déscolarisent pour entrer dans la vie active alors qu'ils atteignent un niveau linguistique supérieur à d'autres, peu scolarisés antérieurement et parfois proches de l'illettrisme, qui réussissent ensuite leur diplôme de CAP.

\section{La question du temps et de l'âge}

L'apprentissage du français nécessite du temps. Un niveau B1 est au moins requis pour aspirer à une orientation en filière générale. Ce niveau B1 est accessible plutôt à partir de la deuxième ou troisième année suivant la connaissance antérieure du français et sur la base de compétences initiales satisfaisantes. Les circulaires prennent en compte un décalage de deux ans par rapport à l'âge de référence. On se rend compte que, pour le cas de cette cohorte, cette mesure est profitable, non pas que les plus âgés apprennent moins vite que les plus jeunes, au contraire car ils obtiennent, en moyenne, des résultats meilleurs au test linguistique que leurs cadets, mais, en raison de leur âge et des impératifs d'orientation, ils sont soumis à une plus grande urgence et exigence pour accéder à la voie scolaire qu'ils souhaitent. Le décalage d'âge se retrouve avec le brevet. Certes, sur le plan national, l'âge de passation du brevet de 15 ans pour la très grande majorité des collégiens ${ }^{84}$ et plus l'élève est âgé, moins il parvient à valider le brevet. De façon générale, les études nationales concluent sur l'incidence du retard scolaire ${ }^{85}$ et le redoublement touche plus les enfants de migrants ${ }^{86}$. Pourtant, en ce qui concerne la cohorte, non pas d'enfants de migrants issus

84 Par exemple, à la session de 2014 pour le brevet général, seulement $13 \%$ des candidats avaient plus de 15 ans. DEPP, RERS édition 2015.

85 Aude Vanhoffelen, Les bacheliers du panel 1995 : évolution et analyse des parcours, Note d'information 10-13, Ministère de l'Éducation Nationale, DEPP (Direction de l'Évaluation, de la Prospective et de la Performance), 2010.

86 Jean-Paul Caille et Louis André Vallet, "Les élèves étrangers ou issus de l'immigration dans l'école et le collège français, une étude d'ensemble ", Les dossiers de l'Éducation et formations $d u$ $M E N, 1996, \mathrm{n}^{\circ} 67$. 
de l'immigration mais d'enfants migrants, la tendance s'inverse et on se rend compte que la très grande majorité des élèves admis a plus de 15 ans (21 élèves ayant plus de 16 ou 17 ans, contre 14 de 15 ans ou moins). Ici, le décalage d'âge caractérise aussi les parcours de réussite d'élèves récemment arrivés.

\section{La question de la durée des «besoins éducatifs particuliers »}

Établir une durée unique serait inadapté à l'hétérogénéité des apprenants, ce qui rejoint la conclusion des Inspections Générales : " ces "besoins particuliers" sont propres à chaque élève et ne peuvent être traités par des mesures uniformes dans une durée limitée. ${ }^{87}$ Effectivement, le rythme d'apprentissage n'est pas le même. Il est ralenti suivant la connaissance antérieure du français (les non francophones ont besoin de plus de temps), la durée de scolarisation antérieure (cas des élèves peu scolarisés antérieurement), la qualité de la scolarisation antérieure et le projet d'intégration ainsi que le rapport à l'école, au pays, à la langue, comme nous l'avons évoqué. Parmi les élèves les plus en difficultés, se trouvaient des jeunes qui n'avaient aucun échange authentique avec les élèves natifs francophones.

En revanche, le rythme est accéléré en fonction des compétences scolaires acquises dans le pays d'origine (les élèves en réussite scolaire dans leur pays d'origine progressent plus vite) et de l'âge (les élèves les plus âgés et dans les classes supérieures, obtiennent en moyenne de meilleurs résultats que leurs cadets). Nous constatons une corrélation entre le niveau scolaire préalable et la progression linguistique, de même nous retrouvons la corrélation du niveau linguistique atteint et de l'orientation dans le cursus scolaire. La facilitation liée à la proximité linguistique de la langue d'origine n'est pas systématique et dépend des compétences scolaires initiales.

Les résultats démontrent l'insuffisance d'une année d'apprentissage et l'impossibilité de préconiser une durée unique d'apprentissage. De ce fait, il est plus pertinent de parler d'un niveau linguistique à atteindre. Le niveau minimum qui caractérise les élèves orientés en filière générale correspond au B1 acquis, d'après le CECRL. Accepter le temps nécessaire permet alors d'accompagner au mieux les réussites des élèves récemment arrivés en France, qu'ils aient été peu scolarisés antérieurement puis orientés en CAP ou qu'ils aient été de brillants élèves dans leur pays d'origine et optent pour une filière générale, technologique ou professionnelle. 


\section{Conclusion et perspectives}

À travers cet article, nous avons questionné des représentations sur des rapports identitaires entre la langue et les élèves. Notre approche n’a pas été exhaustive, loin s'en faut. L'accueil de l'Éducation Nationale a évolué au cours de ces dernières décennies, au gré des choix terminologiques dont l'analyse révèle la variation du champ du public (primo-arrivants, enfants nés en France issus de l'immigration, Français d'outre-mer), les types de prise en charge (dispositifs fermés, ouverts, mixtes, absence de dispositifs) et les idéologies qui les sous-tendent (assimilation, intégration, inclusion), les choix didactiques (enseignement des langues d'origine via les ELCO, cours d'autres disciplines que le français, option d'un enseignement de type langue étrangère ou langue de scolarisation ou soutien scolaire) et les approches pédagogiques (place de l'interculturel, de la langue d'origine, pédagogie du projet, simulation globale...). Difficile ici d'envisager tous les paramètres complexes qui lient accueil institutionnel, trajectoires migratoires, caractères individuels, effet enseignants, effets dispositifs, progrès des élèves... En 2009, suite à une enquête de la Direction générale de l'enseignement scolaire (DGESCO), coordonnée notamment par Catherine Klein, il a été possible de mieux connaitre, du point de vue institutionnel, l'organisation de la scolarisation de ces élèves et l'enseignement mis en œuvre dans les dispositifs. Récemment, le nombre de recherches universitaires portant sur ces questions augmentent.

Actuellement, en réponse d'un appel à projet du Défenseur des droits, un projet vient d'être lancé : EVASCOL ${ }^{88}$ s'inscrit dans les problématiques que nous venons d'énoncer. Il étudie les conditions de scolarisation des enfants allophones, en cherchant à repérer les freins et les leviers à un parcours scolaire réussi. L'étude est articulée autour de 3 axes. Le premier concerne la connaissance sur les conditions effectives de scolarisation depuis l'arrivée en France à l'entrée en classe et, éventuellement, dans le dispositif. Le second axe identifie différentes organisations pédagogiques de dispositifs, mises en œuvre par les enseignants. Des exercices sont proposés aux élèves pour repérer les progrès en langue française et en mathématiques, de sorte à obtenir des indicateurs sur les performances ${ }^{89}$. L'axe 3 , quant à lui, cherche davantage à évaluer la qualité de l'intégration des élèves dans leur établissement, à travers le point de vue de l'enfant, son ressenti et son vécu, pour dépasser le point de vue construit par l'adulte. L'association de chercheurs relevant de différentes disciplines (sociologie, anthropologie, sciences politiques, sciences de l'éducation, didac-

88 https://evascol.hypotheses.org/.

89 Catherine Mendonça Dias, "Le rythme d'apprentissage des élèves allophones arrivants : présentation d'un protocole de recherches ", Dialogues et cultures, n 63, septembre 2017. 
tique des mathématiques et du français...) offre l'opportunité de confronter les points de vue tout en révélant la complexité de l'apprentissage dans une approche holistique de l'enfant qui arrive en France.

\section{Catherine Mendonça Dias}

DILTEC EA 2288

DFLE, Université Sorbonne Nouvelle, Paris 3 catherine.mendonca-dias@univ-paris3.fr

\section{Résumé}

Cet article présente des rythmes dans l'appropriation du français par les élèves récemment arrivés en France. Nous questionnons d'abord les terminologies institutionnelles retenues depuis les années 1970. Puis, nous mettons en évidence quelques caractéristiques individuelles liées à la trajectoire migratoire, qui peuvent aussi avoir une incidence sur l'apprentissage. Enfin, nous communiquons des résultats liés au suivi de cohorte de collégiens nouvellement arrivés qui nous donnent des indicateurs sur le rythme d'appropriation de la langue française et interrogent les représentations que l'institution peut en avoir.

\section{Mots-clés}

Français langue seconde (FLS), apprentissage du français, politiques éducatives, élèves nouvellement arrivés.

\footnotetext{
Abstract

This article presents rhythms of French language acquisition by students recently arrived in France. We first question the institutional terminologies chosen since the 1970s. Then, we highlight some individual characteristics related to the migration paths, which may also have an impact on learning. Finally, we report results related to a cohort of new arrivals followed for 3 years, which gives us indicators the French language learning and question the representations that the institution may have on these students.
}

\section{Keywords}

French as an additional language, French learning, educational policies, new arrivals. 